Article

\title{
Online Estimation of Peak Power Capability of Li-Ion Batteries in Electric Vehicles by a Hardware-in-Loop Approach
}

\author{
Rui Xiong *, Hongwen He *, Fengchun Sun and Kai Zhao \\ National Engineering Laboratory for Electric Vehicles, Beijing Institute of Technology, \\ Beijing 100081, China; E-Mails: sunfch@bit.edu.cn (F.S.); mask2005@163.com (K.Z.) \\ * Authors to whom correspondence should be addressed; E-Mails: rxiong@bit.edu.cn (R.X.); \\ hwhebit@bit.edu.cn (H.H.); Tel.: +86-10-6891-4842; Fax: +86-10-6891-4842.
}

Received: 5 April 2012; in revised form: 9 May 2012 / Accepted: 11 May 2012 /

Published: 15 May 2012

\begin{abstract}
Battery peak power capability estimations play an important theoretical role for the proper use of the battery in electric vehicles. To address the failures in relaxation effects and real-time ability performance, neglecting the battery's design limits and other issues of the traditional peak power capability calculation methods, a new approach based on the dynamic electrochemical-polarization (EP) battery model, taking into consideration constraints of current, voltage, state of charge (SoC) and power is proposed. A hardware-in-the-loop (HIL) system is built for validating the online model-based peak power capability estimation approach of batteries used in hybrid electric vehicles (HEVs) and a HIL test based on the Federal Urban Driving Schedules (FUDS) is used to verify and evaluate its real-time computation performance, reliability and robustness. The results show the proposed approach gives a more accurate estimate compared with the hybrid pulse power characterization (HPPC) method, avoiding over-charging or over-discharging and providing a powerful guarantee for the optimization of HEVs power systems. Furthermore, the HIL test provides valuable data and critical guidance to evaluate the accuracy of the developed battery algorithms.
\end{abstract}

Keywords: electrochemical-polarization model; peak power capability; lithium-ion battery; hybrid electric vehicles; hardware-in-loop 


\section{Introduction}

Energy crises, environmental issues and concerns regarding peaking oil production have promoted research into development of various types of new energy vehicles, which has been established as one of the seven strategic emerging industries in China. Electric vehicles (EVs), which include battery electric vehicles (BEVs), hybrid electric vehicles (HEVs) and plug-in hybrid electric vehicles (PHEVs), are the main development subject for new energy vehicles [1-3]. Electric vehicles (EVs) are emerging as important personal transportation options for petroleum displacement and energy diversification. The battery, as a key component, is crucial for the performance features of EVs, such as economy, power performance, security, etc. [4].

The lithium-ion battery is widely used in many fields because of its advantages of high voltage, low self-discharge and long cycle-life. High specific energy, in particular, makes it a promising candidate for EVs. In such applications, a battery management system (BMS) is critical for maintaining optimum battery performance [5]. Accurate peak power estimates are critical in practical applications since it is necessary to determine the power available in the moment to meet the acceleration, regenerative braking and gradient climbing power requirements without fear of over-charging or over-discharging the battery and thus reducing its lifespan. More importantly, accurate online peak power capability estimates for the battery will optimize the relation between the battery capacity and the vehicle's performance, which benefits the vehicle's general potency [6].

Some peak power capability estimation approaches are presented with the development of EVs technology [6-9]. The commonly used method is the hybrid pulse power characterization (HPPC) method proposed by the Idaho National Engineering \& Environmental Laboratory, which is used for determining the static peak power in laboratory environments [7]. However, it is not suitable for estimating the continuous peak currents that are available for the next sample intervals $\Delta t_{\mathrm{s}}$ and neglects design limits like cell current, cell power or the state of charge (SoC) [8]. In order to overcome the drawbacks of the HPPC method, which is not suitable for continuous peak power capability prediction, and neglecting the SoC limits, the voltage-limited method was proposed by Plett [9]. However, the Rint model-based HPPC and voltage-limited method are not suitable for estimating the battery's peak power capability due to the fact that it can hardly simulate the dynamic voltage performance.

Based on an analysis of the traditional methods [8] proposes a dynamic battery power estimation algorithm taking into account design limit constraints on current, voltage and SoC. The authors of [6] propose a model-based dynamic multi-parameter method for peak power capability estimations, and its comparison with other commonly used methods shows it is more useful than others. Unfortunately, the battery is a strongly non-linear and time-variable system because of its complicated electrochemical processes; its state parameters, such as the equivalent internal resistance, open-circuit voltage (OCV) and available capacity, will be changed to some extent due to the influence of the operation environment, aging and other factors, therefore the model parameters identified by offline methods, which the above two peak power estimation methods use, can hardly be used for a long time with high accuracy. Furthermore, the peak power capability estimation accuracy is always strongly dependent on the reliability of the tabulated OCV-SoC data. In addition, the experiments for collecting the tabulated OCV-SoC data are often time-consuming and error-prone, especially for lithium-ion batteries, which have quite flat OCV-SoC curves. Lastly, it is very challenging to test and verify the developed peak 
power capability estimation algorithm in the early development stage due to limited resources and time.

The well known software MATLAB/Simulink of Math-Works has been widely used in dynamic system modeling and simulation of control algorithms, and the MATLAB toolbox xPC Target provides us a rapid prototyping host-target environment to construct a real time control system in the manner of a hardware-in-the-loop (HIL) test. The software xPC Target is a solution for prototyping, testing, and deploying real-time systems using standard PC hardware [10,11]. The HIL test has been extensively used in the automotive industry for component development and rapid prototyping. Usually the target component under development is tested within a modeled system environment that reproduces the conditions under which the component will operate. With system-level requirements taken into consideration, HIL tests significantly reduce the time and costs of system-level integration and troubleshooting later in the development cycle [12]. The work in this paper aims at developing and verifying the online peak power capability estimation using xPC Target for on-board BMS applications.

This paper makes use of a $3.7 \mathrm{~V} / 35 \mathrm{~A} \mathrm{~h} \mathrm{LiMn}_{2} \mathrm{O}_{4}$ lithium-ion cell as research subject. The paper is arranged as follows: Section 2 proposes an online parameter identification algorithm for the dynamic electrochemical-polarization (EP) battery model with the Simulink/xPC software; Section 3 proposes an online dynamic peak power capability estimation algorithm based on the dynamic EP model and designs a HIL test bench; Section 4 carries out the HIL test and evaluates the proposed method for hybrid electric vehicle (HEV) application; last is the conclusion of this paper.

\section{Online Parameters Identification Method}

To estimate the peak power capability of batteries, a dynamic battery model with an online parameter identification method is required and built first. The purpose of the online parameter identification method is to ensure the real-time performance of the model. The model's precision will decrease because of the error in the model's parameters which may vary with the changes of the operation environment, usage, cooling condition, etc.

\subsection{The EP Model}

Various battery models such as the equivalent circuit model and the electrochemical model are now widely used in EV studies. Electrochemical-polarization was proposed in [13] to integrate the description of the relaxation effect and the inner action in the battery model, and [13] shows the EP model has a better performance than equivalent circuit models and electrochemical models. The EP dynamic model is shown in Figure 1, and the electrical behavior of the model can be expressed by Equation (1):

$$
\left\{\begin{array}{l}
U_{\mathrm{t}}=U_{\mathrm{oc}}-U_{\mathrm{p}}-I_{\mathrm{L}} R_{\mathrm{o}} \\
\dot{U}_{\mathrm{p}}=\frac{I_{\mathrm{L}}}{C_{\mathrm{p}}}-\frac{U_{\mathrm{p}}}{C_{\mathrm{p}} R_{\mathrm{p}}} \\
U_{\mathrm{oc}}=K_{0}+K_{1} \ln S o C+K_{2} \ln (1-S o C)
\end{array}\right.
$$


Figure 1. Schematic diagram for the new electrochemical-polarization model.

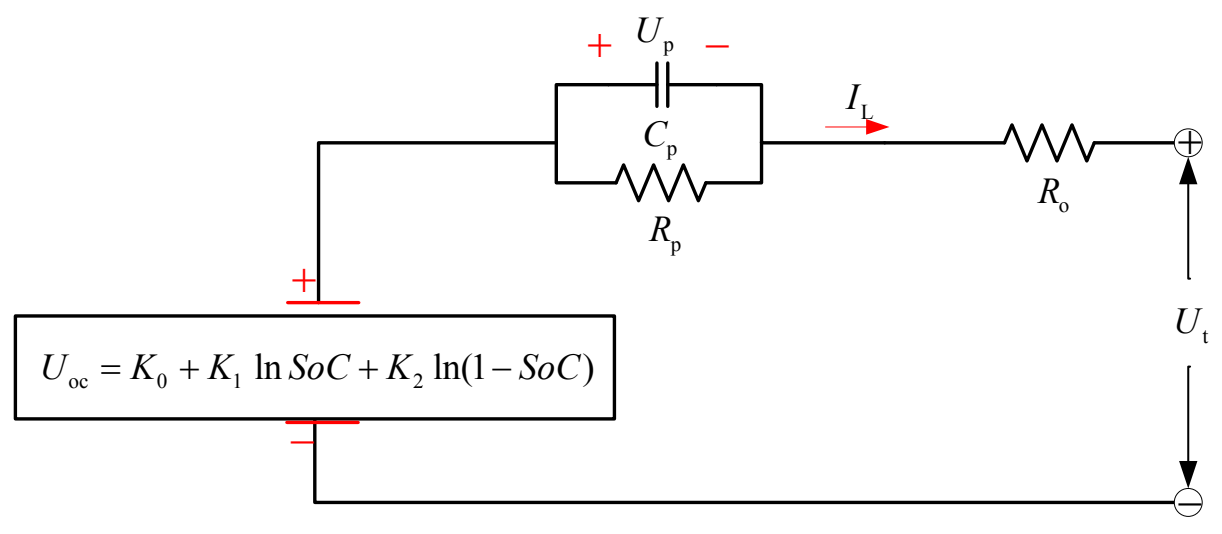

where $K_{0}, K_{1}$ and $K_{2}$ are three constants chosen to make the model well fit the test data, $R_{\mathrm{o}}$ is the ohmic resistance and $R_{\mathrm{p}}$ is the polarization resistance. The polarization capacitance $C_{\mathrm{p}}$ is used to describe the transient dynamic voltage response during charging and discharging. $U_{\mathrm{p}}$ is the polarization voltage across $C_{\mathrm{p}}, U_{\mathrm{t}}$ is the terminal voltage.

\subsection{The Recursive Least Square Method with an Optimal Forgetting Factor}

A model-based method can provide a cheap alternative for estimation or it can be used along with a sensor-based scheme to provide some redundancy. An efficient algorithm is undoubtedly necessary to achieve this goal. The recursive least square (RLS) algorithm is based on the minimization of the sum of squared prediction errors, where the estimated process model parameters are improved progressively with each new process data acquired. The RLS method with an optimal forgetting factor (RLSF) has been widely used in the estimation and tracking of time varying parameters in various fields of engineering. Many successful implementations of RLSF-based adaptive control for time varying parameters estimation are available in the literature $[14,15]$.

Consider a single input single output (SISO) process described by the general higher order auto-regressive exogenous (ARX) model:

$$
y_{\mathrm{k}}=\boldsymbol{\varphi}_{\mathrm{k}}^{\mathrm{T}} \boldsymbol{\theta}_{\mathrm{k}}+\xi_{\mathrm{k}}
$$

where $y$ is the measured system output, which denotes the terminal voltage $U_{\mathrm{t}}$ in this paper. $\boldsymbol{\varphi}$ and $\boldsymbol{\theta}$ are the information matrix and the unknown parameter matrix, respectively. The parameters in $\boldsymbol{\theta}$ can either be constant or subject to infrequent jumps. $\xi$ is a stochastic noise variable (random variable with normal distribution and zero mean), and $\mathrm{k}$ is a non-negative integer, which denotes the sample interval, $\mathrm{k}=0,1,2 \ldots$ For the recursive function of (2), the system identification is realized as follows:

$$
\left\{\begin{array}{l}
\mathbf{K}_{\mathrm{k}}=\frac{\mathbf{P}_{\mathrm{k}-1} \boldsymbol{\varphi}_{\mathrm{k}}}{\lambda+\boldsymbol{\varphi}_{\mathrm{k}}^{\mathrm{T}} k \mathbf{P}_{\mathrm{k}-1} \boldsymbol{\varphi}_{\mathrm{k}}} \\
\mathbf{P}_{\mathrm{k}}=\frac{\mathbf{P}_{\mathrm{k}-1}-\mathbf{K}_{\mathrm{k}} \boldsymbol{\varphi}_{\mathrm{k}}^{\mathrm{T}} \mathbf{P}_{\mathrm{k}-1}}{\lambda} \\
e_{\mathrm{k}}=y_{\mathrm{k}}-\boldsymbol{\varphi}_{\mathrm{k}}^{\mathrm{T}} \hat{\boldsymbol{\theta}}_{\mathrm{k}-1} \\
\hat{\boldsymbol{\theta}}_{\mathrm{k}}=\hat{\boldsymbol{\theta}}_{\mathrm{k}-1}+\mathbf{K}_{\mathrm{k}} e_{\mathrm{k}}
\end{array}\right.
$$


where $\hat{\boldsymbol{\theta}}_{\mathrm{k}}$ is the estimate of the parameter matrix, $e_{\mathrm{k}}$ is the prediction error of the terminal voltage, $\mathbf{K}_{\mathrm{k}}$ is the algorithm gain and $\mathbf{P}_{\mathrm{k}}$ is the covariance matrix, $\lambda$ is the forgetting factor, typically $\lambda=[0.95,1]$ and is very important to obtain a good estimated parameter, set with a small error.

\subsection{The Online Parameters Identification Method for the EP Model}

Equation (1) can be rewritten as follows in the frequency domain:

$$
U_{\mathrm{t}}(s)=U_{\text {oc }}(s)-I_{\mathrm{L}}(s)\left(R_{\mathrm{o}}+\frac{R_{\mathrm{p}}}{1+R_{\mathrm{p}} C_{p} s}\right)
$$

Defining $E_{\mathrm{t}}=U_{\mathrm{t}}-U_{\mathrm{oc}}$, the transfer function $G(\mathrm{~s})$ of Equation (4) can be written as follows:

$$
G(s)=\frac{E_{\mathrm{t}}(s)}{I_{\mathrm{L}}(s)}=-R_{\mathrm{o}}-\frac{R_{\mathrm{p}}}{1+R_{\mathrm{p}} C_{p} s}=-\frac{R_{\mathrm{o}}+R_{\mathrm{p}}+R_{\mathrm{o}} R_{\mathrm{p}} C_{\mathrm{p}} \mathrm{s}}{1+R_{\mathrm{p}} C_{\mathrm{p}} s}
$$

A bilinear transformation method shown in Equation (6) is employed for the discretization calculation of Equation (5) and the result is shown in Equation (7):

$$
s=\frac{2}{T_{\mathrm{s}}} \frac{1-z^{-1}}{1+z^{-1}}
$$

where $z$ is the discretization operator, $T_{\mathrm{s}}$ is the sample intervals and is 1 second for this paper:

$$
G\left(z^{-1}\right)=-\frac{\frac{R_{\mathrm{o}}+R_{\mathrm{p}}+2 R_{\mathrm{o}} R_{\mathrm{p}} C_{\mathrm{p}}}{1+2 R_{\mathrm{p}} C_{\mathrm{p}}}+\frac{R_{\mathrm{o}}+R_{\mathrm{p}}-2 R_{\mathrm{o}} R_{\mathrm{p}} C_{\mathrm{p}}}{1+2 R_{\mathrm{p}} C_{\mathrm{p}}} z^{-1}}{1+\frac{1-2 R_{\mathrm{p}} C_{\mathrm{p}}}{1+2 R_{\mathrm{p}} C_{\mathrm{p}}} z^{-1}}
$$

Define:

$$
\left\{\begin{array}{l}
p_{4}=-\frac{1-2 R_{\mathrm{p}} C_{\mathrm{p}}}{1+2 R_{\mathrm{p}} C_{\mathrm{p}}} \\
p_{5}=-\frac{R_{\mathrm{o}}+R_{\mathrm{p}}+2 R_{\mathrm{o}} R_{\mathrm{p}} C_{\mathrm{p}}}{1+2 R_{\mathrm{p}} C_{\mathrm{p}}} \\
p_{6}=-\frac{R_{\mathrm{o}}+R_{\mathrm{p}}-2 R_{\mathrm{o}} R_{\mathrm{p}} C_{\mathrm{p}}}{1+2 R_{\mathrm{p}} C_{\mathrm{p}}}
\end{array}\right.
$$

The battery's dynamic performance has big relationships with the state parameters as SoC, operating environment, especially the operating temperature $T$, and the usage history $h$. The model parameters can be described as a function of $T$, SoC and $h$. Equation (9) can be simplified to Equation (10) after considering the following assumptions [16]: the expression $\partial S o C / \partial t \approx 0$ holds for battery energy which is consumed or regained in a sample interval relatively small compared to the total capacity available; relying on the proper design of a cooling system/heater for the BMS, the temperature rise/decrease of batteries should be slow, the $\partial T / \partial t \approx 0$ holds for normal operating conditions. The $\partial h / \partial t \approx 0$ definitely holds since $h$ represents a long term usage history: 


$$
\begin{aligned}
\frac{\partial U_{\mathrm{oc}}}{\partial t} & =\left(\frac{K_{1}}{S o C}-\frac{K_{2}}{1-S o C}\right) \frac{\partial S o C}{\partial t} \\
\dot{U}_{\mathrm{oc}} & \approx 0 \Rightarrow U_{\mathrm{oc}, \mathrm{k}} \approx U_{\mathrm{oc}, \mathrm{k}-1}
\end{aligned}
$$

Similarly, $\dot{R}_{\mathrm{o}}=\dot{R}_{\mathrm{o}}(\operatorname{SoC}, \quad T, \quad h)=\frac{\partial R_{\mathrm{o}}}{\partial S o C} \frac{\partial S o C}{\partial t}+\frac{\partial R_{\mathrm{o}}}{\partial T} \frac{\partial T}{\partial t}+\frac{\partial R_{\mathrm{o}}}{\partial h} \frac{\partial h}{\partial t} \approx 0$, since the sample intervals are relatively small, and then:

$$
U_{\mathrm{t}, \mathrm{k}}=p_{1}+p_{2} \ln S o C_{\mathrm{k}}+p_{3} \ln \left(1-S o C_{\mathrm{k}}\right)+p_{4} U_{\mathrm{t}, \mathrm{k}-1}+p_{5} I_{\mathrm{L}, \mathrm{k}}+p_{6} I_{\mathrm{L}, \mathrm{k}-1}
$$

where:

$$
\left\{\begin{array}{l}
p_{1}=K_{0}\left(1-p_{4}\right) \\
p_{2}=K_{1}\left(1-p_{4}\right) \\
p_{3}=K_{2}\left(1-p_{4}\right)
\end{array}\right.
$$

Then the model parameters can be solved by the expressions of $p_{1}, p_{2}, p_{3}, p_{4}, p_{5}, p_{6}$, and as follows:

$$
\left\{\begin{array}{l}
R_{\mathrm{o}}=\frac{p_{6}-p_{5}}{1+p_{4}}, R_{\mathrm{p}}=-\frac{1}{4} \frac{p_{6}+p_{4} p_{5}}{p_{4}^{2}-1}, C_{\mathrm{p}}=2 \frac{\left(p_{4}+1\right)^{2}}{p_{6}+p_{4} p_{5}} \\
K_{0}=\frac{p_{1}}{1-p_{4}}, K_{1}=\frac{p_{2}}{1-p_{4}}, K_{2}=\frac{p_{3}}{1-p_{4}}
\end{array}\right.
$$

Define $\boldsymbol{\varphi}_{\mathrm{k}-1}=\left[\begin{array}{lllllll}1 & \ln S o C_{\mathrm{k}} & \ln \left(1-S o C_{\mathrm{k}}\right) & U_{\mathrm{t}, \mathrm{k}-1} & I_{\mathrm{Lk}} & I_{\mathrm{L}, \mathrm{k}-1}\end{array}\right], \boldsymbol{\theta}_{\mathrm{k}-1}=\left[\begin{array}{llllll}p_{1} & p_{2} & p_{3} & p_{4} & p_{5} & p_{6}\end{array}\right]^{\mathrm{T}}$ and $y_{\mathrm{k}-1}=U_{\mathrm{t}, \mathrm{k}-1}$. In case of an online application, herein the $U_{\mathrm{t}, \mathrm{k}}$ and $I_{\mathrm{L}, \mathrm{k}}$ are sampled at constant periods, the vector $\boldsymbol{\theta}_{\mathrm{k}}$ can be identified by a recursive least squares algorithm according to Equation (3), and then the model parameters can be solved by the expressions of $p_{1}, p_{2}, p_{3}, p_{4}, p_{5}, p_{6}$.

\section{Battery Online Peak Power Capability Estimation Method}

To estimate the battery's peak power capability, an online EP dynamic model-based peak power capability estimation approach is proposed and built with the Simulink software first. Afterwards, this section will deal with the design of a hardware-in-loop test bench for the evaluation of the proposed method.

\subsection{Online Peak Power Capability Estimation Method}

\subsubsection{The HPPC Method}

The HPPC method, developed by Idaho National Engineering \& Environmental Laboratory [7], estimates the peak power capability of a battery based on the Rint model consisting of an OCV and an ohmic resistance. A cell is simulated by the following relation:

$$
U_{\mathrm{t}}(t)=U_{\mathrm{oc}}(s(t))-R_{\mathrm{o}} \times I_{\mathrm{L}}(t)
$$

where $U_{\mathrm{t}}(t)$ is the terminal voltage at time $t ; U_{\mathrm{oc}}(s(t))$ the open-circuit voltage at present SoC state $(s(t))$, and $R_{\mathrm{o}}$ the charging or discharging internal resistance. 
Due to the design limit $U_{\mathrm{t}, \min } \leq U_{\mathrm{t}}(t) \leq U_{\mathrm{t}, \max }$, the peak charge and discharge current under the voltage constraints are described as:

$$
\left\{\begin{array}{l}
I_{\mathrm{min}, \mathrm{k}}^{\mathrm{chg}, \mathrm{HPC}}=\frac{U_{\mathrm{oc}, \mathrm{k}}-U_{\mathrm{t}, \text { max }}}{R_{\mathrm{o}}} \\
I_{\mathrm{max}, \mathrm{k}}^{\mathrm{dis}, \mathrm{HPC}}=\frac{U_{\mathrm{oc}, \mathrm{k}}-U_{\mathrm{t}, \text { min }}}{R_{\mathrm{o}}}
\end{array}\right.
$$

where $U_{\mathrm{t}, \max }$ and $U_{\mathrm{t}, \min }$ are the maximum limit voltage when charging and the minimum voltage limit when discharging, respectively; $I_{\min }^{\mathrm{chg}, \mathrm{HPPC}}, I_{\max }^{\mathrm{dis}, \mathrm{HPPC}}$ are the minimum charge current and maximum discharge currents based on the HPPC method. Hence, the peak power capability of the lithium-ion cell can be described as:

$$
\left\{\begin{array}{l}
P_{\min }^{\mathrm{chg}}=U_{\mathrm{t}, \max } \times I_{\min }^{\mathrm{chg}, \mathrm{HPPC}} \\
P_{\text {max }}^{\mathrm{dis}}=U_{\mathrm{t}, \text { min }} \times I_{\text {max }}^{\mathrm{dis}, \mathrm{HPC}}
\end{array}\right.
$$

This method can be used for calculating the absolute available peak power capability of the battery pack, but it is not suitable for estimating the continuous available peak power capability at the next $\Delta t$. It takes into account the operational design limits on voltage when estimating maximum available power, but does not consider design limits on cell current, cell power or cell SoC.

\subsubsection{The SoC-Limited Method}

With the SoC-limited method, the peak current, which is used for estimating the battery available peak power capability, can be obtained based on the maximum and minimum SoC limits. Starting from time $t$, the battery is discharged (or charged) with a constant current during the specified time period $\Delta t$. For the time period $\Delta t$, the SoC of the cell at the time $(t+\Delta t)$ can be expressed by:

$$
s(t+\Delta t)=s(t)-I_{\mathrm{L}}(t)\left(\frac{\eta_{\mathrm{i}} \Delta t}{C_{\max }}\right)
$$

where $\eta_{\mathrm{i}}$ is a coulomb efficiency factor for the current level $I_{\mathrm{L}}(t)$, which is in function of the load current. $C_{\max }$ is the present maximum available capacity.

Due to the consideration of the design limits of HEVs: the maximum SoC $s_{\max }$, the minimum SoC $s_{\min }$ and the current limit of cell can be expressed as follows:

$$
\left\{\begin{array}{l}
I_{\min }^{\mathrm{chg}, \mathrm{soc}}=\frac{s(t)-s_{\max }}{\eta_{\mathrm{i}} \Delta t / C_{\max }} \\
I_{\max }^{\mathrm{dis}, \mathrm{soc}}=\frac{s(t)-s_{\min }}{\eta_{\mathrm{i}} \Delta t / C_{\max }}
\end{array}\right.
$$

The SoC-limited method which focuses on the continuous peak power capability within time $\Delta t$ conforms to the practical charge and discharge process. However, this method will give optimistic estimations of the battery's peak current if the battery is allowed to be discharged or charged over a wide range of SoC. As a result, this method is usually applied together with another method. 


\subsubsection{The EP Dynamic Model-Based Method}

The HPPC method is widely used for the calculation of the battery's peak power capability in laboratories. However, the Rint model is so simple that it can only be used to do power predictions under stable driving cycles; and it can hardly be used for HEVs since their battery operation environment is very harsh. The above mentioned method is therefore not appropriate for estimating the battery's peak power capability in HEVs. To solve these problems, the EP model-based dynamic peak power capability estimation method is proposed. The model can precisely simulate the dynamic polarization characteristics of a lithium ion battery and obtain the ideal prediction.

Discretization of Equation (1):

$$
U_{\mathrm{p}}(t+\Delta t)=\exp (-\Delta t / \tau) U_{\mathrm{p}}(t)+R_{\mathrm{p}}(1-\exp (-\Delta t / \tau)) I_{\mathrm{L}}(t)
$$

However, the peak currents cannot be solved directly from the maximum current $I_{\mathrm{L}}(t)$ since $s(t+\Delta t)$ itself is a function of the current $I_{\mathrm{L}}(t)$ and OCV is a nonlinear function of $s(t)$. Concerning this problem, the Taylor-series expansion is employed to linearize the equation and to solve the approximated values for the peak currents. The Taylor-series expansion equation is as follows:

$$
U_{\text {oc }}(s(t+\Delta t))=U_{\text {oc }}\left(s(t)-I_{\mathrm{L}}(t) \frac{\eta_{\mathrm{i}} \Delta t}{C_{\max }}\right)=U_{\text {oc }}(s(t))-\left.I_{\mathrm{L}}(t) \frac{\eta \Delta t}{C_{\max }} \frac{\partial U_{\mathrm{oc}}(s)}{\partial s}\right|_{s=s(t)}+R_{1}\left(s(t), I_{\mathrm{L}}(t) \frac{\eta_{\mathrm{i}} \Delta t}{C_{\max }}\right)
$$

Considering the first-order residual $R_{1}(\bullet)$ being too small to affect the OCV at the next $\Delta t$, since the SoC's variation per sample interval is very small [16,17], the $R_{1}(\bullet)$ can be viewed as $R_{1}(\bullet) \approx 0$. Then:

$$
\left\{\begin{array}{c}
I_{\max }^{\mathrm{dis}, \text { The }}=\frac{U_{\mathrm{oc}}(s(t))-U_{\mathrm{p}}(t) \exp \left(-\frac{\Delta t}{\tau}\right)-U_{\mathrm{t}, \text { min }}}{\left.\frac{\eta_{\mathrm{i}} \Delta t}{C_{\max }} \frac{\partial U_{\mathrm{oc}}(s)}{\partial s}\right|_{s(t)}+R_{\mathrm{p}}\left[1-\exp \left(-\frac{\Delta t}{\tau}\right)\right]+R_{\mathrm{o}}} \\
I_{\min }^{\mathrm{chg}, \text { hhe }}=\frac{U_{\mathrm{oc}}(s(t))-U_{\mathrm{p}}(t) \exp \left(-\frac{\Delta t}{\tau}\right)-U_{\mathrm{t}, \max }}{\left.\frac{\eta_{\mathrm{i}} \Delta t}{C_{\max }} \frac{\partial U_{\mathrm{oc}}(s)}{\partial s}\right|_{s(t)}+R_{\mathrm{p}}\left[1-\exp \left(-\frac{\Delta t}{\tau}\right)\right]+R_{\mathrm{o}}}
\end{array}\right.
$$

where $\partial U_{\text {oc }}(s) / \partial s=K_{1} / s-K_{2} /(1-s)$ can be obtained from Equation (1). The OCV value is going up as the SoC increases, as a result the $\partial U_{\text {oc }}(s) / \partial s$ is positive for most batteries within the entire SoC operation range. The polarization voltage $U_{\mathrm{p}}$ is greater than zero when the battery is discharging and $U_{\mathrm{p}}<0$ when charging, therefore the values computed by (21) are smaller in magnitude than those from (15) for the same ohmic resistance values. Meanwhile, $\partial U_{\mathrm{oc}} / \partial \mathrm{s}$ is not constant within the entire SoC operation range, especially at the two extreme ends. Therefore the peak power capability estimates based on the EP-model method are more reasonable than those of the HPPC method regarding peak current calculations and thus safety issues. Once the current design limit is calculated, the peak currents with all limits enforced are calculated as:

$$
\left\{\begin{array}{l}
I_{\max }^{\mathrm{dis}}=\min \left(I_{\max }, I_{\max }^{\mathrm{dis}, \mathrm{SoC}}, I_{\max }^{\mathrm{dis}, \mathrm{EP}}\right) \\
I_{\min }^{\mathrm{chg}}=\max \left(I_{\min }, I_{\min }^{\mathrm{chg}, \mathrm{SoC}}, I_{\min }^{\mathrm{chg}, \mathrm{EP}}\right)
\end{array}\right.
$$


where $I_{\max }, I_{\min }$ are the cell's current design limits, $I_{\max }$ denotes the maximum discharge current and $I_{\min }$ denotes the minimum charge current of its design limits. The peak power capability may be calculated as follows:

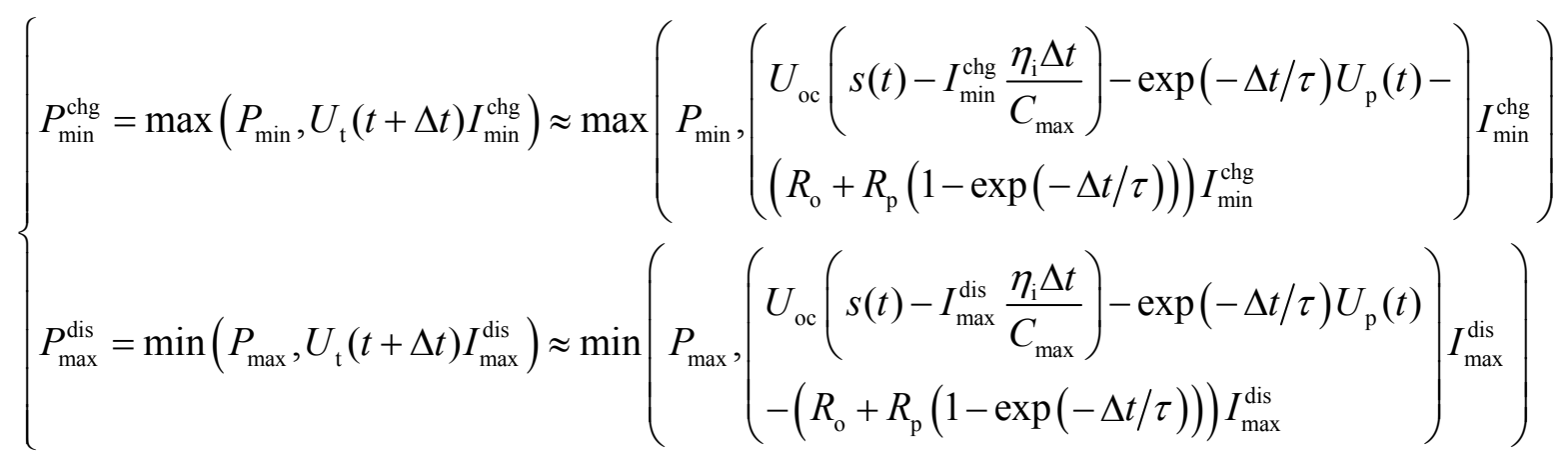

where $P_{\max }, P_{\min }$ are the cell's power design limits, $P_{\max }$ denotes the peak discharge power and $P_{\min }$ denotes the peak charge power of its design limits. Then we can build the Simulink model of the peak power capability estimation methods for the HPPC method and the EP dynamic model-based method, respectively, based on the equations above.

\subsection{The Hardware-in-Loop Test Bench Design}

The test bench for the HIL test is shown in Figure 2, which consists of electrical load equipment, a CAN communication unit, a host computer and Target. The electrical load equipment can charge/discharge a battery according to the real-time demands of the current or power with a maximum voltage of $50 \mathrm{~V}$ and a maximum charging/discharging current of $200 \mathrm{~A}$. Its recorded data include current, voltage, temperature, accumulative Amp-hours (A h) and Watt-hours (W h). The errors of the Hall current and voltage sensors are less than $0.2 \%$ and $0.5 \%$, respectively. The measured data is transmitted to the Target through CAN. Both the Target and the electrical load equipment have a low-pass filtering function to implement large noise cancellation.

The software is running on two PCs, the host PC and the Target PC. The host PC runs the Microsoft Windows XP operating system and the required software packages: MATLAB, Simulink, Real-Time Workshop (RTW) and $\mathrm{C} / \mathrm{C}++$ compiler. MATLAB is the host software environment, which includes Simulink with XPC Target and RTW module. In this environment, a desktop or laptop computer is used as the host PC with MATLAB and Simulink to create a model using Simulink blocks. After creating the model with Simulink, the simulation can be run first. Afterwards, xPC Target allows us to add I/O blocks to the model, and then use the host PC with Real-Time workshop and a $\mathrm{C} / \mathrm{C}++$ compiler to create an executable code. The executable code is downloaded from the host PC to the target PC running the xPC Target real-time kernel, which is booted from a floppy disk. The communication between the host and target PC is established through TCP/IP. After downloading the executable code, we can run and test the target application in real time. In order to reduce the influence of temperature, the battery is carried out in a thermal chamber with a fixed temperature of $20^{\circ} \mathrm{C}$. 
Figure 2. The test bench for HIL experiment.

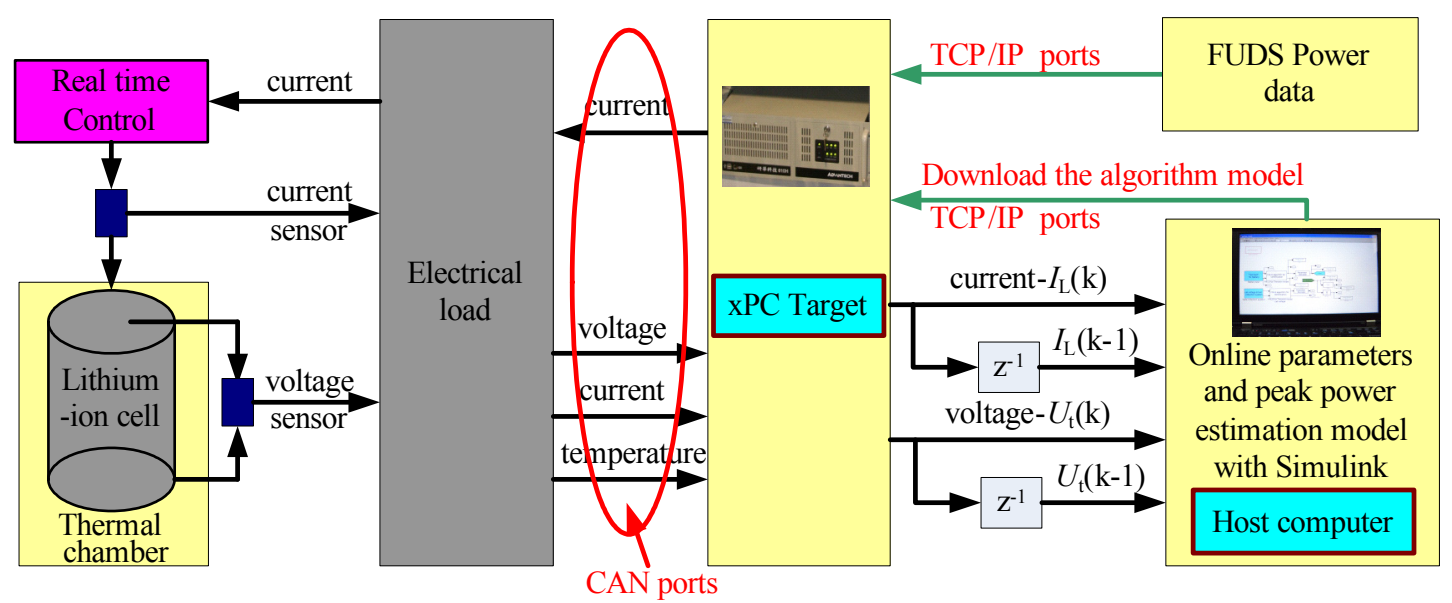

\section{Results and Discussion}

Firstly, the cell capacity check test is run to check the cell's present maximum available capacity. After being properly initialized (the cell's design limits are shown in Table 1), the battery then runs through a verification profile of the Federal Urban Driving Schedules (FUDS) $[18,19]$ on the HIL bench downloaded from the xPC Target. The measured current and voltage profiles of the FUDS are shown in Figure 3. To simplify the analysis for the peak power capability calculation results, the SoC is calculated by the ampere counting method, which is useful in a laboratory environment. The initial SoC is set at 0.85 . The battery model takes measured voltages, currents, and temperatures of the battery from HIL testing as inputs, and calculates the $\mathrm{SoC}$ as an input for the model and estimates the power capabilities over time.

Table 1. The cell's design limits for the HIL test.

\begin{tabular}{cc}
\hline Parameters & Value \\
\hline Maximum load current /A & 350 \\
Minimum load current /A & 175 \\
Maximum terminal voltage /V & 4.2 \\
Minimum terminal voltage /V & 3.0 \\
Peak discharge power /W & 1500 \\
Peak charge power /W & -700 \\
SoC operation range for HEVs & $0.35-0.85$ \\
\hline
\end{tabular}

Figure 3. The measured profiles of the FUDS cycles: (a) current; (b) voltage.
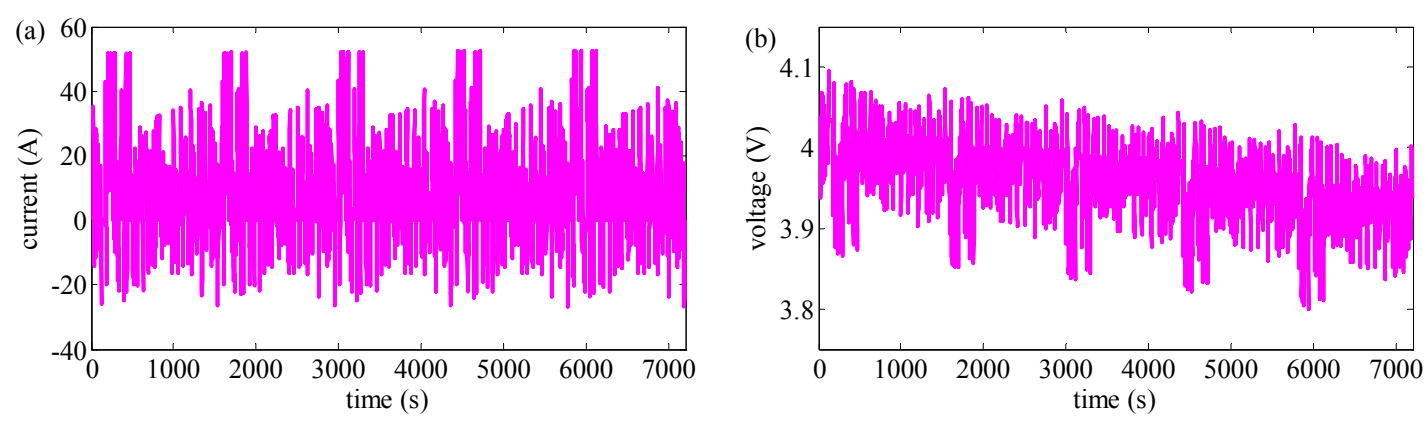
The online parameters identification results for parameter matrix $\theta_{\mathrm{k}}$ are shown in Figure 4 . The model's parameters can be deduced by Equation (13) and the results are shown in Figure 5.

Figure 4. The online identification results for parameter matrix $\boldsymbol{\theta}_{\mathrm{k}}$ : (a) $p_{1} \sim p_{4}$; (b) $p_{5}$ and $p_{6}$.
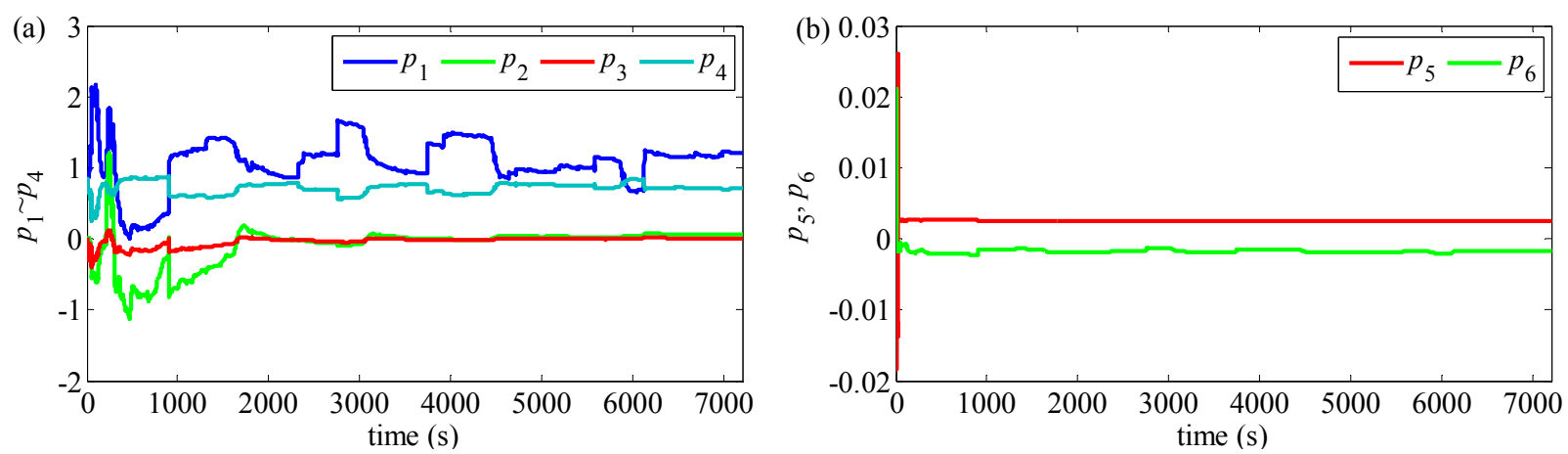

Figure 5. Online parameters identification results of the EP model and calculated SoC profiles: (a) $K_{0}, K_{1}, K_{2}$; (b) Calculated SoC; (c) $U_{\mathrm{oc}} ;$ (d) $R_{\mathrm{o}} ;(\mathbf{e}) R_{\mathrm{p}} ;(\mathbf{f}) C_{\mathrm{p}}$.
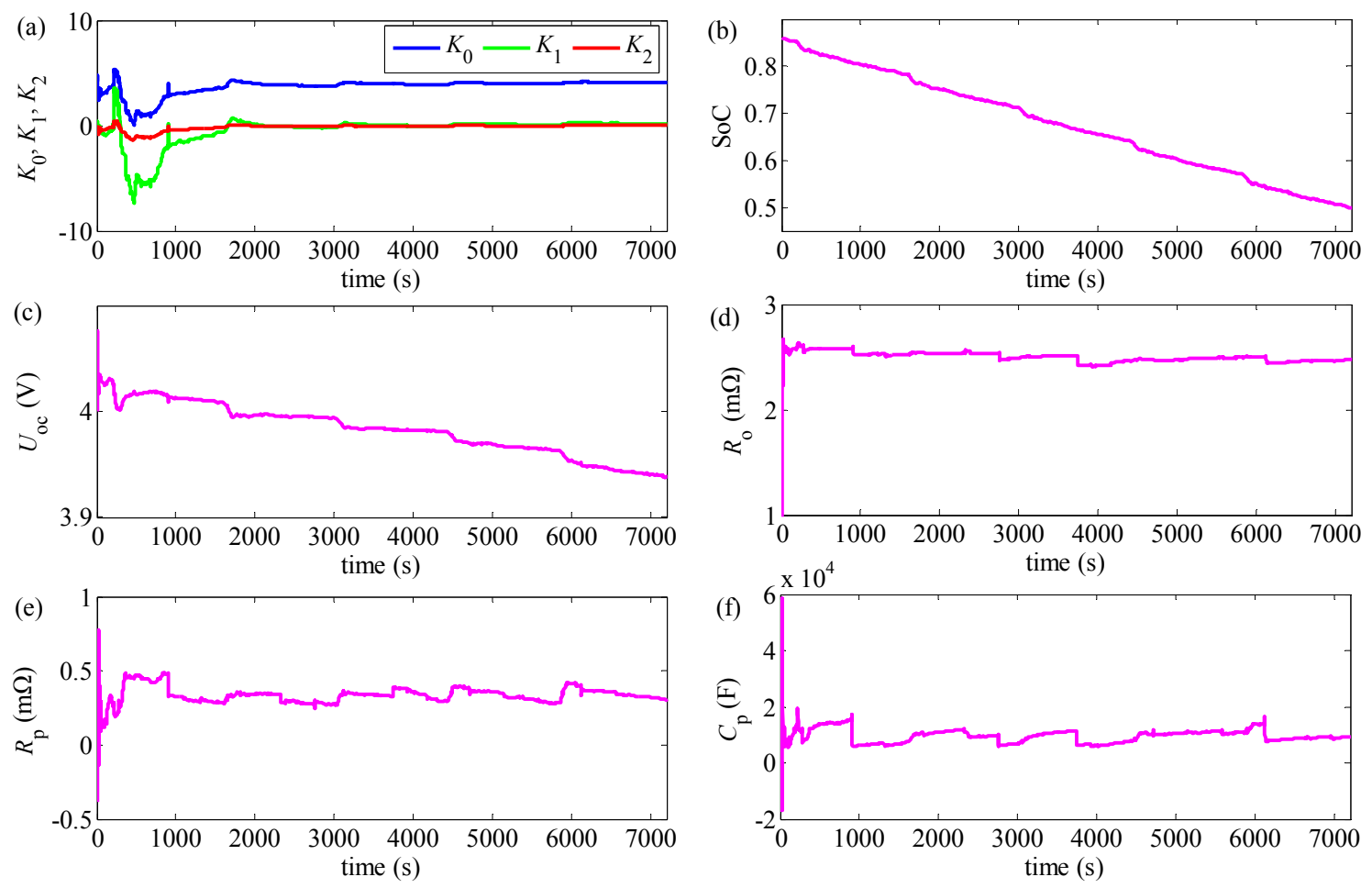

Then we can evaluate, for the online parameters identification method, the accuracy of the estimated terminal voltage. Figure 6 shows the online estimated terminal voltage error and its statistical information. In Figure 6(a), it is shown that with the RLSF-based online parameters identification approach, good accuracies can be obtained with the maximum estimated voltage error being smaller than $0.03 \mathrm{~V}$ (within $1 \%$ of its nominal voltage which is $0.037 \mathrm{~V}$ ), which suggests the EP model has good dynamic performance. The mean estimated error is usually the preferred evaluation index since it takes into account each individual observation and is most amenable to statistical analysis, and Figure 6(b) shows the maximum mean error is less than $1 \mathrm{mV}$, and the mean error is not always decreasing as time goes on, but has small amplitude variations, which occur because an 
accurate estimate depends on the simulation precision of the operating environment and process noise, the proposed online identification algorithm requires update the estimates and decrease the estimation errors continuously.

Figure 6. The comparison profiles of the terminal voltages: (a) The voltage error between the measured value and the online estimated value; (b) Error's statistical information.
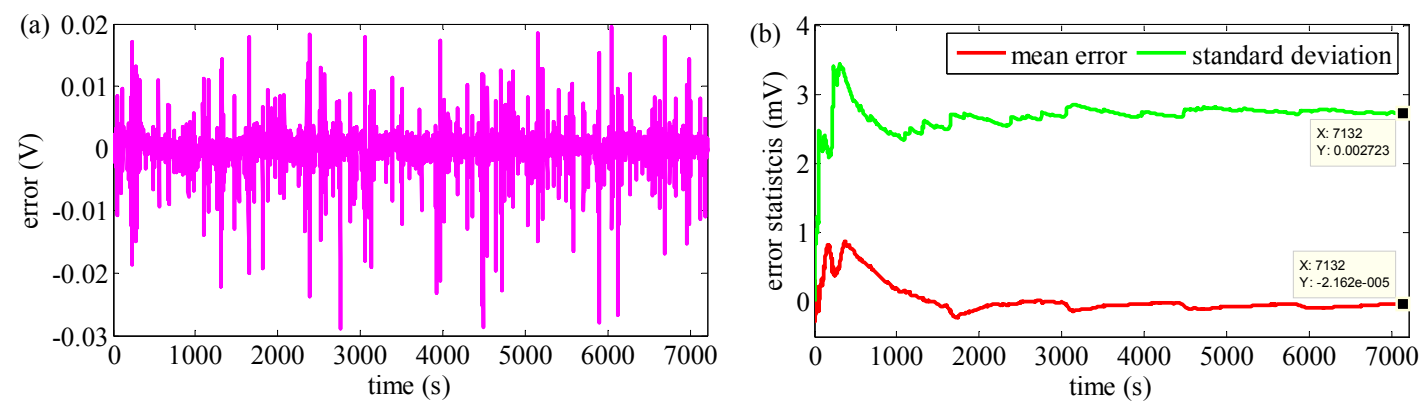

The mean error of estimated voltage is near zero, which indicates the feasibility of the online parameter identification method. Further, Figure 6(b) shows the standard deviation of estimated error which is a measure of how spread out the observation values is, which is less than $3.7 \mathrm{mV}$, which enhance the evidence of the efficiency and the reliability performance of the proposed approach. More importantly, the errors converge to the measured value quickly, which indicates the accuracy of the EP model is enough for other battery calculation algorithms, and the RLSF algorithm has better predictive abilities of the uncertain variables if the time-consuming experiments for periodical parameters calibration are avoided.

The real-time peak power capability and current estimation results are shown in Figure 7. Figure 7(a) shows that the peak discharge current calculated with the HPPC method is higher than with the EP dynamic model-based method. A notable feature of the EP dynamic model-based method is that it takes the entire $\partial U_{\mathrm{oc}} / \partial \mathrm{s}>0$ of the cell into account when making a prediction while the former doesn't. For $\partial U_{\mathrm{oc}} / \partial \mathrm{s}>0$, which leads to a lower peak discharge current value in contrast to the values of the HPPC method, the result is a more realistic representation. Further, the EP dynamic model can simulate the dynamic effect of lithium-ion batteries well, while the Rint model fails to do this. When discharging, $U_{\mathrm{p}}>0$, it is obvious that the current based on the EP model is lower, especially in the ranges of high required power. The estimated peak current with the proposed peak power capability estimation decreases quickly because the polarization voltage increases quickly. Figure 7(b) shows the peak currents with the EP dynamic model-based method, which, considering the lithium-ion battery relaxation effect is well simulated, can meet the actual performance well. If the SoC value reaches the maximum design limit, the peak charge current will be very small while the peak discharge current will be very big. On the contrary, if the SoC value reaches the minimum design limit, the peak discharge current will be very small while the peak charge current will be very big. As a result, the method can optimize the operation range of batteries and extend their lifespan. At the same time, when the battery is being discharged with big currents, the peak discharge value is reduced significantly while the peak charge capability is significantly increased. This is conforming to the actual battery working characteristics and underlying optimization control. 
Figure 7. Peak power capability and current online estimation results: (a) Peak discharge currents; (b) Peak charge currents; (c) Peak discharge powers; (d) Peak charge powers.
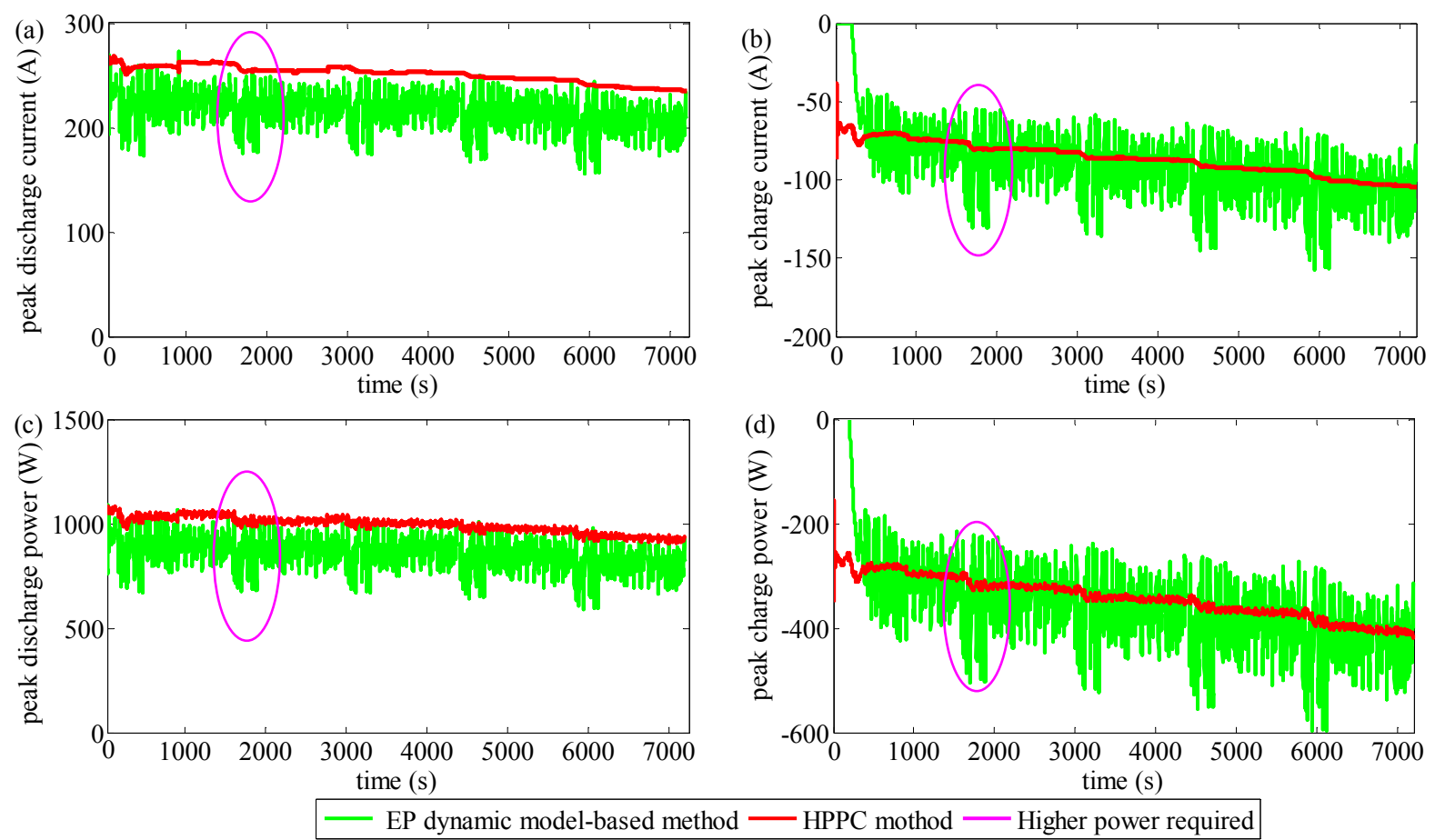

Figure 7(c) shows that the peak discharge power calculation with the HPPC method is obviously higher than the calculation with the proposed method. This is because the HPPC method only considers the battery voltage constraints, neglecting the constraints of the cell current, SoC and power. Furthermore this method gives an optimistic estimation and may lead to over-discharging. A notable feature of the proposed new method is that it takes the entire dynamics of the cell into account when making real-time estimations. The strong discharges at the high required power ranges pull the cell's terminal voltage down, allowing less discharge power than with the HPPC method. So the HPPC method overpredicts the charge power at high SoC. However, the biggest problem of the HPPC method comes from ignoring the SoC design limits. It also overpredicts power when a continuous big current is required, while it ignores the dynamic performance from the polarization voltage $U_{\mathrm{p}}$. The two methods are also compared with respect to charging power, as shown in Figure 7(d). Due to the ignorance of the SoC limits and the relaxation effect of the battery, the HPPC method is prone to overpredicting the charging power. With strong discharges at high required power ranges, the battery will allow a greater charging power while the HPPC method cannot give the accuracy peak power estimates quickly to match the driving cycle changes. However, the real-time peak power capability estimation results with the EP dynamic model-based method are not flat due to the polarization effects. Therefore the proposed method for peak power capability estimation gives satisfying results.

Overall, the HIL test suggests that the proposed online peak power capability estimation is reliable, and can give more accurate estimation results than the traditional methods. Furthermore, the online parameter identification method can ensure the performance of the EP dynamic model. More importantly, the proposed peak power capability estimation can be applied at electric vehicles without requiring a periodical calibration for ensuring the model's accuracy. 


\section{Conclusions}

A battery HIL test system was implemented and employed to develop and validate the battery peak power capability estimation approach for a $\mathrm{LiMn}_{2} \mathrm{O}_{4}$ lithium-ion cell. Based on the above analysis, the following main concluding remarks can be made:

(1) In order to avoid time-consuming, laborious and error-prone experiments for determining the tabulated OCV-SoC data, the EP model, which uses the Nernst model to define the open circuit voltage, is applied to model the lithium-ion battery.

(2) For improving the dynamic performance of the EP model, the RLSF algorithm is applied to identify online the EP model's parameters; the model's accuracy is verified by the hardware-in-loop test, and the maximum error of the estimated terminal voltage is within $1 \%$ of its nominal voltage.

(3) Compared with the HPPC method, the proposed peak power capability estimation method takes the cell voltage, current, SoC and power as its constraints; which can simulate the relaxation effect well. The evaluation results based on the HIL test show that the proposed method gives a more reliable estimation, especially when the load current changes suddenly or strongly. More importantly, when the SoC is high and low, the proposed method can give a more accurate estimate; avoiding overcharging or overdischarging.

(4) The HIL test data has provided critical guidance for further development and improvement of the peak power capability estimation approach. This accelerates the overall system development process and reduces the cost of the EVs development.

Our future work will focus on combining the online state estimation and peak power capability estimation in order to improve the prediction accuracy and the applicability of the model-based BMS.

\section{Acknowledgments}

This work was supported partly by the National High Technology Research and Development Program of China (2011AA112304, 2011AA11A228, 2011AA1290), partly by the International Cooperation Research Program of Chinese Ministry of Science and Technology (2010DFB70090) and also by the Beijing Institute of Technology Post Graduate Students Innovation Foundation. The author would also like to thank the reviewers for their corrections and helpful suggestions.

\section{References}

1. Su, M.; Liang, C.; Chen, B.; Chen, S.; Yang, Z. Low-carbon development patterns: Observations of typical Chinese cities. Energies 2012, 5, 2913-2904.

2. He, H.W.; Xiong, R.; Chang, Y.H. Dynamic modeling and simulation on hybrid power system for electric vehicle application. Energies 2010, 3, 1821-1830.

3. He, H.W.; Yan, S.; Xiao, Z. Integrated control method for a fuel cell hybrid system. Asia-Pac. J. Chem. Eng. 2009, 4, 68-72.

4. He, H.; Xiong, R.; Fan, J. Evaluation of lithium-ion battery equivalent circuit models for state of charge estimation by an experimental approach. Energies 2011, 4, 5825-5898. 
5. Kim, J.; Lee, S.; Cho, B.H. Discrimination of Li-ion batteries based on hamming network using discharging-charging voltage pattern recognition for improved state-of-charge estimation. J. Power Sources 2011, 196, 2227-2240.

6. Sun, F.; Xiong, R.; He, H.; Li, W.; Aussems, J. Model-based dynamic multi-parameter method for peak power estimation of lithium-ion batteries. Appl. Energy 2012, 96, 3773-3785.

7. Idaho National Engineering \& Environmental Laboratory. Battery Test Manual for Plug-In Hybrid Electric Vehicles; Assistant Secretary for Energy Efficiency and Renewable Energy (EE) Idaho Operations Office: Idaho Falls, ID, USA, 2010.

8. Zhang, C.; Zhang, C.; Sharkh, S. Estimation of real-time peak power capability of a traction battery pack used in an HEV. In Proceedings of the Asia-Pacific Power and Energy Engineering Conference (APPEEC), Chengdu, China, 2010; pp.1-6.

9. Gregory, L. High-performance battery-pack power estimation using a dynamic cell model. IEEE Trans. Veh. Tech. 2004, 53, 15861-15893.

10. Low, K.; Wang, H.; Wang, M. On the development of a real time control system by using xPC target: Solution to robotic system control. In Proceedings of the 2005 IEEE International Conference on Automation Science and Engineering, Edmonton, Canada, 1-2 August 2005; pp. 3453-3450.

11. Shiakolas, P.; Piyabongkarn, D. On the development of a realtime digital control system using xPC target and magnetic levitation device. In Proceedings of the International Conference on Decision and Control, Orlando, FL, USA, 2001; pp. 1384-1353.

12. He, Y.; Liu, W.; Brain, J. Battery algorithm verification and development using hardware-in-the-loop testing. J. Power Sources 2010, 195, 2969-2974.

13. Xiong, R.; He, H.; Guo, H.; Ding, Y. Modeling for lithium-ion battery used in electric vehicles. Procedia Eng. 2011, 15, 2869-2874.

14. Goodwin, G.C.; Sin, K.S. In Adaptive Filtering, Prediction and Control; Prentice-Hall: Upper Saddle River, NJ, USA, 1984.

15. Ho, Y.; Farouq, S.; Yeoh, H. Recursive least squares-based adaptive control of a biodiesel transesterification reactor. Ind. Eng. Chem. Res. 2010, 49, 11434-11442.

16. Yi-Hsien, C.; Wu-Yang, S.; Jia-Cheng, K. Online estimation of internal resistance and opencircuit voltage of lithium-ion batteries in electric vehicles. J. Power Sources 2011, 196, 3921-3932.

17. He, H.; Xiong, R.; Guo, H. Online estimation of model parameters and state-of-charge of $\mathrm{LiFePO}_{4}$ batteries in electric vehicles. Appl. Energy 2012, 89, 413-420.

18. Kruse, R.; Huls, T. Development of the federal urban driving schedule. SAE Tech. Pap. 1973, doi: $10.4271 / 730553$.

19. Zhang, C.P.; Jiang, J.C.; Zhang, W.G.; Suleiman, M.S. Estimation of state of charge of lithium-ion batteries used in HEV using robust extended Kalman filtering. Energies 2012, 5, 10981-11115.

(C) 2012 by the authors; licensee MDPI, Basel, Switzerland. This article is an open access article distributed under the terms and conditions of the Creative Commons Attribution license (http://creativecommons.org/licenses/by/3.0/). 